\title{
Species-dependent variations in the dielectric activity of membrane skeleton of erythrocytes
}

\author{
Ivan T. Ivanov ${ }^{1}$, Boyana K. Paarvanova ${ }^{1}$, Bilyana B. Tacheva ${ }^{1}$ and Todor Slavov ${ }^{2}$ \\ ${ }^{1}$ Department of Physics, Biophysics, Roentgenology and Radiology, Medical Faculty, Thracian University, Stara Zagora, \\ Bulgaria \\ ${ }^{2}$ Department of Animal Morphology, Physiology and Nutrition, Faculty of Agriculture, Thracian University, Stara Zagora, \\ Bulgaria
}

\begin{abstract}
Previously detected $\beta_{\mathrm{sp}}$ and $\gamma 1_{\mathrm{sp}}$ dielectric relaxations on the spectrin-based membrane skeleton (MS) of human red blood cells (RBCs) have been shown sensitive to the attachment of MS to the lipid-protein membrane. Such relaxations were now detected on the MS of mammal (rat, horse, bovine, sheep and goat) and "unstrained" chicken RBCs. To become "unstrained" chicken RBCs were subjected consecutively to cold $\left(4^{\circ} \mathrm{C},>20 \mathrm{~h}\right)$ and either colchicine $(15 \mathrm{mM})$ or vinblastine $(30 \mu \mathrm{M})$ $\left(4^{\circ} \mathrm{C}, 1 \mathrm{~h}\right)$ that led to irreversible disassembly of their marginal band and an additional portion of their cytoskeleton. With the exception of bovine RBCs, the critical frequency $\left(f_{c}\right)$ of either relaxation increased, although at different rates, with the decrease in the volume of RBC species. The strong increase in $f_{\mathrm{c}}$ of $\gamma 1_{\text {sp }}$ relaxation from $2.5 \mathrm{MHz}$ ("unstrained" chicken RBCs) to $13 \mathrm{MHz}$ (goat RBCs) could indicate denser state of MS in smaller RBC species. The low values of $f_{\mathrm{c}}$ of $\gamma 1_{\mathrm{sp}}$ relaxation in "unstrained" chicken RBCs $(2.5 \mathrm{MHz})$ and bovine RBCs ( 4.5 instead of $9 \mathrm{MHz}$ ) could be related to their extraordinary thermal stability at the temperature of spectrin denaturation.
\end{abstract}

Key words: Chicken erythrocyte - Mammal erythrocyte - Membrane skeleton - Dielectric relaxation - Spectrin

\begin{abstract}
Abbreviations: RBC, red blood cell; MS, spectrin-based membrane skeleton; $f$, frequency of alternating electric field; $f_{c}$, characteristic frequency of dielectric relaxation; $f_{\beta s p}$, characteristic frequency of $\beta_{\text {sp }}$ relaxation; $f_{\gamma 1 s p}$, characteristic frequency of $\gamma 1_{\text {sp }}$ relaxation; $T_{\mathrm{A}}$, denaturation temperature of $\mathrm{RBC}$ spectrin.
\end{abstract}

\section{Introduction}

Compared to the biconcave mammal red blood cells (RBCs), the bird RBCs look like flattened ovoids and have about five to seven times lower deformability and elasticity (Waugh and Evans 1976). Major differences between mammal and bird RBCs are the cytoskeleton and cellular nucleus, which are present in the latter cells and absent in the former cells. Nevertheless, mammal and bird RBCs have almost identical plasma membranes containing

Correspondence to: Ivan T. Ivanov, Department of Physics, Biophysics, Roentgenology and Radiology, Medical Faculty of Thracian University, Stara Zagora 6000, Bulgaria

E-mail: ivanov_it@gbg.bg lipid-protein membrane and a spectrin-based protein submembrane skeleton (MS).

The MS of chicken RBCs (Granger et al. 1982) and human RBCs (Saldanha and Oliveira 2010) includes the interwoven filaments of the single major peripheral protein spectrin with foci composed of actin oligomers. Spectrin is long (maximum about $200 \mathrm{~nm}$ ) filamentous protein that constitutes about $30 \%$ of membrane protein mass (Liu et al. 1990) and is attached to the cytoplasmic surface of the lipid-protein membrane by two types of high-affinity protein bridges. The first bridge (attachment site) includes actin oligomer, bound to the first major integral protein glycophorin $\mathrm{C}$, while the second bridge contains ankyrin which connects spectrin to the tetramers of band 3, the last major integral protein (Fig. 1). The basic elements of 


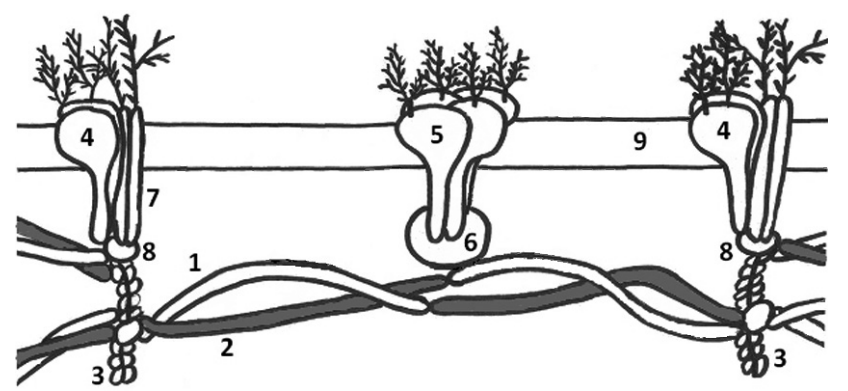

Figure 1. A schematic diagram of human RBC membrane: alpha spectrin monomer (1), beta spectrin monomer (2), actin olygomer (3), band 3 dimer (4), band 3 tetramer (5), ankyrin (6), glycophorin C (7), band 4.1 (8), lipid bilayer (9). Adapted from Tse and Lux (1999).

erythrocyte MS and its attachment to the lipid-protein membrane seem to be remarkably similar from species to species (Whitfield et al. 1983).

The cytoskeleton of bird RBCs is composed of microtubules, actin microfilaments and intermediate filaments which demonstrate strong structural interdependence. The disruption of one filament system frequently affects the integrity of others (cross-talk). Intermediate filaments contain chiefly vimentin and synemin and span between the nucleus and MS with ankyrin and spectrin being their chief plasma membrane docking sites (Granger et al. 1982; Georgatos and Marchesi 1985). Microfilaments contain mainly polymerized actin in two forms, stress fibers and sub-plasmalemmal actin cortex (Salbreux et al. 2012; Clark et al. 2013).

Microtubules contain polymerized tubulin and form hooplike sub-plasmalemmal bundle (marginal band) that encircles the cell like a narrow belt confined to the periphery of the cell (Glomski and Pica 2011). In adult chicken RBCs the microtubules are completely refractile to high concentrations of microtubule depolymerizing drugs (e.g. colchicine and vimentin) but demonstrate reversible cold sensitivity (Miller and Solomon 1984; Kim et al. 1987). They completely depolymerize in cells incubated at $4^{\circ} \mathrm{C}$ for $1 \mathrm{~h}$ and re-form precisely after consequent re-warming $\left(39^{\circ} \mathrm{C}, 10 \mathrm{~min}\right)$. The cold-induced depolymerization of marginal band of chicken RBCs has been reported to eliminate the elasticity of these cells although preserving their disc-shape (Glomski and Pica 2011).

In human, plenty of serious diseases are associated with the worsening of mechanical function (elasticity, deformability and stability) of human RBCs (Marchesi 2008). The stability and deformability of human RBCs highly depend on the strength of above mentioned attachment sites (Anong et al. 2009) while the cytoskeleton and nucleus could impair the deformability of chicken RBCs. Compared to chicken RBCs the segmental flexibility and attachment of MS in human RBCs are much better studied. Human RBC spectrin exhibits considerable segmental motion with a correlation time $\sim 10^{-6} \mathrm{~s}$ which strongly contributes to the mechanical function of MS (Fung et al. 1996).

The dynamics of macromolecules is frequently studied by the method of dielectroscopy. In biological tissues and cell suspensions the strong dielectric relaxation taking place within the radio frequency range $(0.1-10 \mathrm{MHz})$ is due to the charging up (Maxwell-Wagner effect) of plasma membranes of cells (structural or interfacial or $\beta$ ) and orientation of the net permanent dipoles of proteins (molecular dipolar or $\gamma 1$ relaxation) (Schwan 1981). The dipolar dielectric relaxation arises when interaction between alternating electric field and a group of identical electric dipoles becomes reduced at a frequency, $f_{\mathcal{C}}$, characteristic for these dipoles (Gimsa 2017). Dipolar relaxations involving polar side chains of proteins $(\gamma 2)$, bound water $(\gamma 3)$ and free water $(\gamma)$ are placed at much higher frequencies. Compared to the $\beta$, the $\gamma 1$ relaxation of proteins has much smaller strength (dielectric increment) and almost the same $f_{\mathrm{c}}$. For example, the $\gamma 1$ relaxation of hemoglobin is about $1 \mathrm{MHz}$ (Simeonova et al. 2002). Another contribution to the $\beta$ relaxation is caused by the subcellular organelles. Since these structures are smaller in size than the surrounding cell, their $f_{c}$ are higher but their total dielectric increment smaller (Schwan 1981; Asami 2015).

The dielectric activity of MS in human RBCs was recently studied by differential thermal dielectroscopy (Ivanov and Paarvanova 2016, 2019a). According to Brandts et al. (1977), spectrin of human RBC sustains sharp denaturation at the temperature of $49.5^{\circ} \mathrm{C}$, further denoted as $T_{\mathrm{A}}$. No major membrane and cytosol proteins denature close to $T_{\mathrm{A}}$. At the same temperature, frequency dependent threshold changes in the complex impedance, $\Delta Z^{*}$, and complex capacitance, $\Delta C^{*}$, of suspensions containing human RBCs, human RBC ghost membranes and isolated MSs were detected (Ivanov and Paarvanova 2016). Considerable evidence was presented that these changes were related to the alteration of dielectric properties of human RBC plasma membrane consequent to the denaturation of spectrin. The frequency analysis of above mentioned $\Delta Z^{\star}$ and $\Delta C^{\star}$ values, based on the methods of dielectric spectroscopy (Klösgen et al. 2011), revealed two single-time dielectric relaxations (Ivanov and Paarvanova 2016, 2019a). As the major alteration of membranes at $T_{\mathrm{A}}$ was the denaturation of spectrin, whose dielectric activity at denatured state was nil, the above mentioned $\Delta Z^{*}$ and $\Delta C^{\star}$ changes and respective dielectric relaxations were ascribed to the contribution of the native spectrin to the dielectric properties of human RBC membranes.

The $f_{\mathrm{c}}$ of the first relaxation on MS (further called $\beta_{\mathrm{sp}}$ relaxation) coincided with the $f_{\mathrm{c}}$ of the structural $\beta$ relaxation on lipid-protein membranes, when the latter was shifted up and down by changing the ion concentration of suspension medium. The left shoulder of $\beta_{\mathrm{sp}}$ relaxation occupied the low frequency interval (1-100 kHz) whereat the RBC cyto- 
sol was effectively isolated from outside field. In addition, the strength of $\beta_{\text {sp }}$ relaxation was strongly reduced after weakening the interaction between MS and lipid-protein membrane, predominantly the spectrin-actin junction (Ivanov and Paarvanova 2019a). Based on above results, the $\beta_{\text {sp }}$ relaxation was assumed related to a direct piezoeffect on the flexible spectrin filaments powered by the electrostriction of lipid-protein membrane. The second (further called $\gamma 1_{\mathrm{sp}}$ ) relaxation had $f_{\mathrm{c}}$ of about $7 \mathrm{MHz}$ and was detected in a frequency interval whereat the field penetrated cytosol and interacted directly with the spectrin dipoles. Similar relaxation has been reported as a slight tail of the powerful $\beta$ relaxation on the plasma membrane of human RBCs and assumed as a dipolar relaxation of some (membrane) proteins (Schwan and Takashima 1993; Martinsen et al. 2002).

In this study, the same approach was used to compare the dielectric properties of MS in enucleated erythrocytes from mammals (human, rat, horse, bovine, sheep and goat) with those in nucleated erythrocytes from birds (chicken). In all species the erythrocyte MS demonstrated similar $\beta_{\mathrm{sp}}$ relaxation and similar $\gamma 1_{\text {sp }}$ relaxation. In contrast to mammal RBCs, chicken RBCs had to be "unstrained" in order to demonstrate dielectric activity on their MS. The "unstraining" conditions included sufficiently long cooling $(20 \mathrm{~h}$ at $\left.4^{\circ} \mathrm{C}\right)$ with consequent treatment $\left(1 \mathrm{~h}\right.$ at $\left.4^{\circ} \mathrm{C}\right)$ by colchicine $(15 \mathrm{mM})$ or vinblastine $(30 \mu \mathrm{M})$. With the exception of bovine RBCs, the $f_{\mathrm{c}}$ of either relaxation tended to increase, although at different rates, with the decrease in the volume of RBC species. While the effect of cell volume on the $f_{\mathrm{c}}$ of $\beta_{\mathrm{sp}}$ relaxation could be consequence of the well-known similar cell volume-induced effect on the $f_{\mathrm{c}}$ of $\beta$ relaxation on the lipid membrane, the effect of cell volume on the $f_{\mathrm{c}}$ of $\gamma 1_{\mathrm{sp}}$ relaxation could indicate that the smaller was the volume of $\mathrm{RBC}$ species the more contracted and dense was their MS.

\section{Materials and Methods}

\section{Materials}

$\mathrm{NaCl}$, mannitol, colchicine and vinblastine were purchased from Sigma Chemicals Co, St. Louis, MO, USA.

\section{Isolation of $R B C s$}

Chicken, human, rat, horse, bovine, sheep and goat RBCs were isolated by centrifugation $(250 \times g$, $5 \mathrm{~min})$ from freshly drawn heparinized blood. The upper layer of white blood cells was discarded. Isolated RBCs were washed once in excess volume of $150 \mathrm{mM} \mathrm{NaCl}$ and two times in excess volume of isotonic solution containing $10 \mathrm{mM} \mathrm{NaCl}$ and $280 \mathrm{mM}$ mannitol (conductivity $0.11 \mathrm{~S} / \mathrm{m}$ ) further called working medium. Except otherwise indicated, the studied RBCs were suspended in the working medium at hematocrit value of 0.55 , determined by microhematocrit capillaries method. To prepare RBC ghost membranes, the washed human RBCs were lysed hypotonically and the obtained plasma membranes resealed in $75 \mathrm{mM} \mathrm{NaCl}\left(37^{\circ} \mathrm{C} 20 \mathrm{~min}\right.$ ) (Ivanov and Paarvanova 2016). The RBC ghost membranes were isolated, washed in medium of $5 \mathrm{mM} \mathrm{NaCl}$ and $140 \mathrm{mM}$ mannitol and suspended in the same medium at hematocrit 0.55 . The prepared suspensions of RBCs and RBC ghost membranes were subjected to test heating across $T_{\mathrm{A}}$.

\section{Cold-induced depolymerization of chicken RBC marginal band}

Prior to test heating, the microtubules of chicken RBCs were depolymerized. The reversible depolymerization was obtained incubating washed and packed chicken RBCs at $4^{\circ} \mathrm{C}$ for time intervals up to $30 \mathrm{~h}$. To preserve the depolymerized state of microtubules during test heating, the cold-treated cells were further incubated in a cool working medium, containing either colchicine $(15 \mathrm{mM})$ or vinblastine $(30 \mu \mathrm{M})$, hematocrit 0.55 at $4^{\circ} \mathrm{C}$ for one hour. In case the cold-treatment lasted more than $20 \mathrm{~h}$, the cells with irreversibly depolymerized marginal band were conventionally called "unstrained".

\section{Differential thermal dielectroscopy of $R B C$ suspensions at $T_{\mathrm{A}}$}

To conduct the test heating, $70 \mu \mathrm{l}$ of suspension, hematocrit 0.55 , was introduced with a syringe into a conductometric cuvette. The latter was made up of a glass tube (length $120 \mathrm{~mm}$, outside diameter $4 \mathrm{~mm}$, wall thickness $0.5 \mathrm{~mm}$ ) containing two platinum electrodes spaced at $3 \mathrm{~mm}$. The cuvette was tightly inserted in a hole, drilled into an aluminum block whose temperature was controlled by a thermocouple. To denature spectrin the block was heated with constant heating rate $\left(1.5^{\circ} \mathrm{C} / \mathrm{min}\right)$ across the $T_{\mathrm{A}}\left(53.5^{\circ} \mathrm{C}\right.$ for chicken RBCs, $49.5^{\circ} \mathrm{C}$ for human RBCs and about $50^{\circ} \mathrm{C}$ for rat, horse, bovine, sheep and goat RBCs). The $T_{\mathrm{A}}$ values, indicated in the text, are corrected by the effect of heating rate. The flat ovoid shape of both intact and "unstrained" chicken RBCs practically does not changed at $T_{\mathrm{A}}$ (Paarvanova et al. 2014; see Fig. 2). On suspension in low salt medium, e.g. in the working medium, human RBCs obtain stomatocytic shape at room temperature while at $T_{\mathrm{A}}$ they reportedly obtain cup (i.e., stomatocytic) form (Eskelinen et al. 1985). In our test heating this insignificant shape change at $T_{\mathrm{A}}$ was accompanied by insignificant fragmentation and vesiculation due to the high rate of heating. After prolong heating at the spectrin denaturation temperature $\left(49^{\circ} \mathrm{C}, 10 \mathrm{~min}\right)$, bovine erythrocytes have also been shown to obtain stomatocytic shape without any visible fragmentation or vesiculation of 


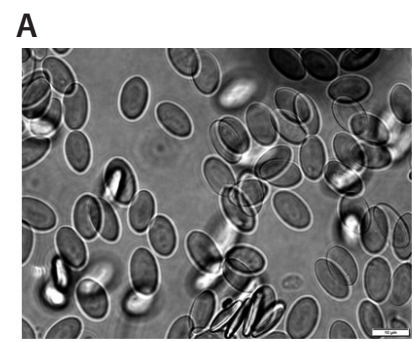

c

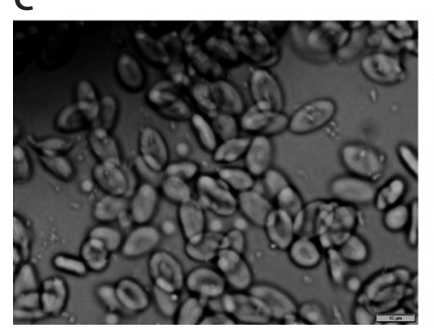

B

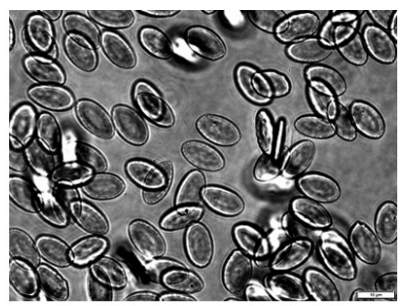

D

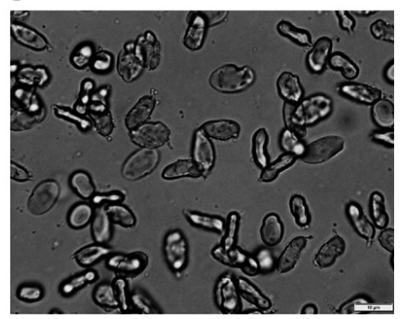

Figure 2. Micrographs of washed chicken RBCs: cells at room temperature (A); cells, heated at $56^{\circ} \mathrm{C}$ for $5 \mathrm{~min}(\mathrm{~B})$; cells cooled at $4^{\circ} \mathrm{C}$ with $15 \mathrm{mM}$ colchicine for interval of 2 to $20 \mathrm{~h} \mathrm{(C)}$; "unstrained" cells, cooled at $4^{\circ} \mathrm{C}$ with $15 \mathrm{mM}$ colchicine for $20 \mathrm{~h}$ and heated at $56^{\circ} \mathrm{C}$ for $4 \mathrm{~min}(\mathrm{D})$. Bar indicates $10 \mu \mathrm{m}$.

their membrane (Mosior et al. 1990). Based on above data and taking into account the immense number and random orientation of suspended RBCs, the shape factor was regarded not important for the values of dielectric changes at $T_{\mathrm{A}}$, as early reported (Ivanov and Popov 1993).

The complex impedance, $Z^{*}=Z^{\prime}+j$. $Z^{\prime \prime}$, admittance, $Y^{*}=$ $Y^{\prime}+j$. $Y^{\prime \prime}$, and capacitance, $C^{*}=C^{\prime}-j$. $C^{\prime \prime}$, of tested suspension were continuously measured and separated into their real $(Z$, $\left.Y^{\prime}, C^{\prime}\right)$ and imaginary ( $\left.Z^{\prime \prime}, Y^{\prime \prime}, C^{\prime \prime}\right)$ parts using Solartron 1260 Impedance Frequency Analyzer (Schlumberger Instruments, Hampshire, England) controlled by a computer. Here, $j$ is the imaginary unit, $j^{2}=-1$. The $Z^{\star}, Y^{\star}$ and $C^{\star}$ values were measured at 16 frequencies between $20 \mathrm{kHz}$ and $15 \mathrm{MHz}$, and scanned sequentially with an integration time of $0.5 \mathrm{~s}$.

Within the frequency interval of $\beta$ relaxation the real capacitance, $C$, of human $\mathrm{RBC}$ suspensions is chiefly determined by the capacitance of plasma membrane. At low frequencies the latter is maximal and equal to the static capacitance of plasma membrane while it strongly decreases about the $f_{\mathrm{c}}$ of $\beta$ relaxation. The imaginary capacitance, $C$ ", (also called dissipation) represents the power at which the field irreversibly dissipates energy by rotating the dipoles (dielectric loss, $C_{\mathrm{d}}$ ") and moving the free ions (conductive loss). The latter loss was determined and subtracted from $C$ " in order to obtain $C_{\mathrm{d}}$ ”, as explained next.

At a fixed temperature, $C^{\prime \prime}$ of the suspension medium is purely conductive and the $\log \left(C^{\prime \prime}\right) / \log (f)$ plot represents a slant straight line over the frequency interval of $20 \mathrm{kHz}-$ $15 \mathrm{MHz}$ (Gabriel et al. 1996). Similarly, at low frequencies ( $f$ between $20 \mathrm{kHz}$ and $100 \mathrm{kHz}$ ) the conductive loss in tested suspensions strongly prevailed over the dielectric loss and the $\log \left(C^{\prime \prime}\right)$ linearly declined with $\log (f)$ (Fig. 3A). Above $500 \mathrm{kHz}$ however, the dielectric loss prevailed and the linear shape of $\log \left(C^{\prime \prime}\right) / \log (f)$ dependence was disturbed. The mathematical fit (the power-law is preferable to linear) of the low frequency linear portion $(20 \mathrm{kHz}-40 \mathrm{kHz})$ was obtained and used to extrapolate the conduction loss data to higher frequencies. The shadowed area between the ex-
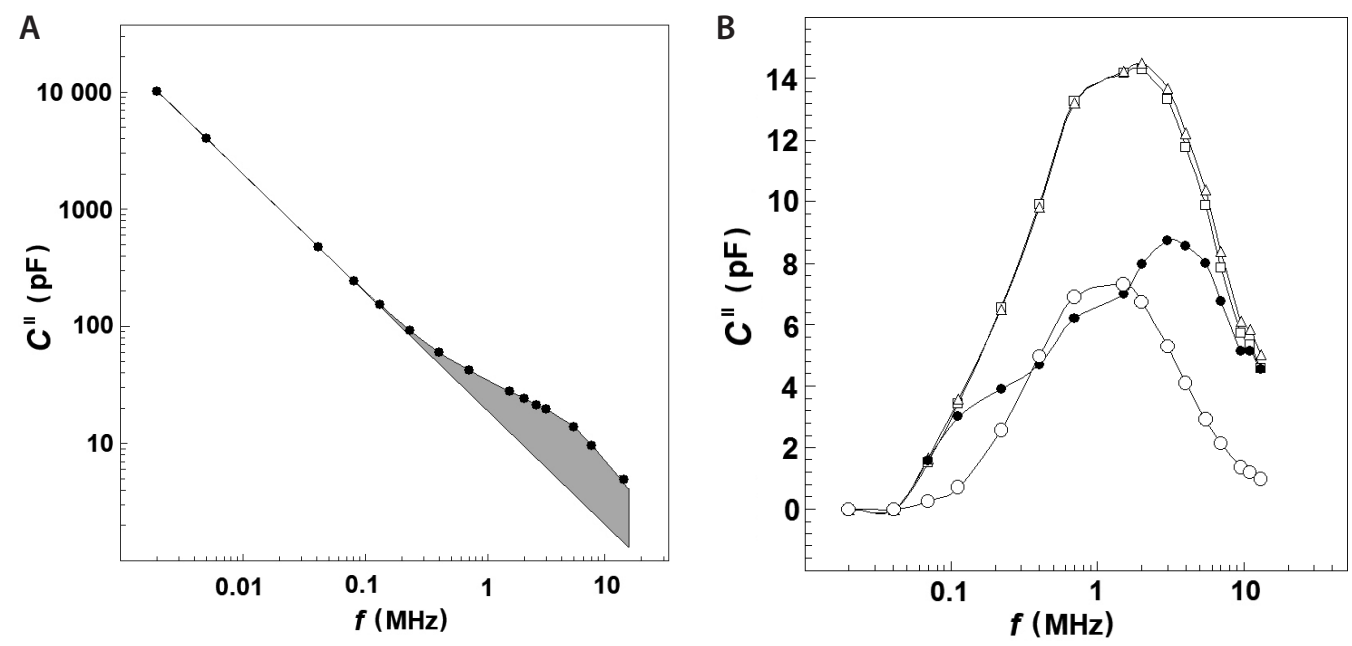

Figure 3. A. Effect of frequency on the electric energy dissipated in suspension of either human RBCs or their ghost membranes. The energy loss is expressed by the imaginary impedance, $C^{\prime \prime}(\mathrm{pF})$, of suspension plotted against the frequency of electric field, $f(\mathrm{MHz})$. The temperature and hematocrit were $25^{\circ} \mathrm{C}$ and 0.55 , respectively. The straight line corresponds to the conduction loss in sus-

pension while shadowed area represents the dielectric loss curve, $C_{\mathrm{d}}$ " $(\mathrm{pF})$, of plasma membranes. B. Temperature dependence of the frequency curve of dielectric loss dissipated in suspensions of either human RBCs or their ghost membranes. The dielectric loss is expressed by $C_{\mathrm{d}}$ " $(\mathrm{pF})$, plotted against the frequency of electric field, $f(\mathrm{MHz})$ (shadowed area in $\mathrm{A}$ ). The temperature of suspension was $41^{\circ} \mathrm{C}(\square), 47^{\circ} \mathrm{C}(\Delta)$ and $53^{\circ} \mathrm{C}(\bullet)$. The hematocrit was 0.55 . The temperature-corrected differential dielectric loss curve at $\mathrm{T}_{\mathrm{A}}$ is noted by $(O)$. 
trapolated straight line and experimental curve represented the frequency dependent $C_{\mathrm{d}}$ ", the dielectric loss curve of suspension at the given temperature.

The polarization of electrodes impedes the precise measurement of suspension capacitance, $C^{\prime}$, at low frequencies (Wolf et al. 2011). It was reduced using platinum electrodes, low inter electrode voltage $(75 \mathrm{mV})$, high frequencies, high hematocrit value $(0.55)$ and suspension medium with low ionic strength $(10 \mathrm{mM} \mathrm{NaCl})$ and low conductivity. Mannitol was preferred to sucrose due to its lower thickening ability. To check the effect of electrode polarization below $0.5 \mathrm{MHz}$, control experiments were carried out with another cuvette, containing two low polarizable parallel-plate, platinized platinum electrodes with area of $0.6 \mathrm{~mm}^{2}$, displaced at $2 \mathrm{~mm}$. The electrodes were mounted into rectangular glass vessel submerged into externally heated machine oil. Based on the results obtained with two cuvettes we came to the conclusion that some data for the $C^{\star}$ of intact cells and cells with denatured spectrin $\left(C^{\prime}\right.$ below $40 \mathrm{kHz}$ and $\mathrm{C}^{\prime}$ below $20 \mathrm{kHz}$ ) are not reliable and were excluded.

\section{Temperature corrections of the apparent dielectric changes in heated suspensions at $T_{A}$}

As previously noted (Ivanov and Paarvanova 2016), all dielectric parameters, $Z$, $Z$ ', $Y^{\prime}, Y^{\prime \prime}, C^{\prime}$, and $C^{\prime \prime}$ of heated RBC suspensions exhibited sharp, frequency dependent, sigmoid changes induced by the denaturation of spectrin at $T_{A}$. For $Y$, measured at a fixed frequency, this is exemplified at Fig. 4. The magnitude of the apparent threshold change in $Y^{\prime}$ at $T_{A}$ was defined as $\left(\Delta Y^{\prime}\right)_{\text {apparent }}=\left(Y^{\prime}\right)_{\mathrm{n} 2}-\left(Y^{\prime}\right)_{\mathrm{n} 3}$, where $\left(Y^{\prime}\right)_{\mathrm{n} 2}$ and $\left(Y^{\prime}\right)_{\mathrm{n} 3}$ are the real part of the admittance at the native state of spectrin (at a temperature $2^{\circ} \mathrm{C}$ less than $T_{A}$ ) and at the denatured state of spectrin (at a temperature $4^{\circ} \mathrm{C}$ greater than $T_{A}$ ), respectively. This change, however, levels off within a substantial temperature interval of $6^{\circ} \mathrm{C}$ where it is superimposed on the temperature variations of a multitude of processes involving the plasma membrane, suspension medium and cytosol (ion conductivity of media, molecular dipolar relaxations of cytosol proteins, $\beta$ relaxations of cell organelles ect), all assayed at the indicated frequency. As the temperature interval is very short, these temperature variations could be considered small and linear because they are not caused by protein denaturation. Hence, to eliminate latter variations on temperature, which had no relation to the huge threshold change in $Y^{\prime}$ at $T_{A}$, the variation in $Y^{\prime}$, taking place over an equal temperature interval prior to $T_{A}$, i.e., $\left(Y^{\prime}\right)_{\mathrm{n} 1}-\left(Y^{\prime}\right)_{\mathrm{n} 2}$, was determined and subtracted from the $\left(\Delta Y^{\prime}\right)_{\text {apparent }}$. Therefore, at the indicated frequency the corrected change in $Y^{\prime}$ at $T_{A}$ was expressed as $\Delta Y^{\prime}=\left[\left(Y^{\prime}\right)_{\mathrm{n} 2}\right.$ $\left.-\left(Y^{\prime}\right)_{\mathrm{n} 3}\right]-\left[\left(Y^{\prime}\right)_{\mathrm{n} 1}-\left(Y^{\prime}\right)_{\mathrm{n} 2}\right]=2\left(Y^{\prime}\right)_{\mathrm{n} 2}-\left(Y^{\prime}\right)_{\mathrm{n} 1}-\left(Y^{\prime}\right)_{\mathrm{n} 3}$ (see Fig. 4). The corrected changes in $Z, Z$ ", $Y^{\prime \prime}, C^{\prime}$, and $C_{\mathrm{d}}$ " at $T_{A}$, i.e. $\Delta Z, \Delta Z^{\prime \prime}, \Delta C^{\prime}, \Delta Y^{\prime \prime}$ and $\Delta C_{\mathrm{d}}$ " were likewise obtained for each one of all tested frequencies. The temperature correction of the differential dielectric loss curve at $T_{A}, \Delta C_{\mathrm{d}}$ ", is shown at Fig. $3 \mathrm{~B}$ and the obtained curve (depicted by the open circles) had a bell shape. This and all next figures represent the typical result from multiple experiments.

\section{Results}

\section{Acquisition of raw data}

The threshold changes in complex admittance and complex capacitance at $T_{\mathrm{A}}$, obtained with heated suspensions of either human RBCs or resealed human RBC ghost membranes, were corrected for the continuous effect of temperature on the accompanying non-threshold processes and noted as $\Delta Y^{\prime}(f), \Delta Y^{\prime \prime}(f), \Delta C^{\prime}(f)$ and $\Delta C_{\mathrm{d}}$ " $(f)$, where $\Delta C_{\mathrm{d}}$ " $(f)$ represents the dielectric component of $\Delta C^{\prime \prime}(f)$. These changes were almost identical when obtained with either human RBCs or resealed human RBC ghost membranes. This finding, combined with the applied temperature correction, indicated that the changes in dielectric parameters at $T_{\mathrm{A}}$ were dominated chiefly by the denaturation of the major membrane protein spectrin and subsequent threshold alteration of plasma membranes. Based on the assumption that first, the dielectric activity of denatured spectrin is nil within the frequency interval from $20 \mathrm{kHz}$ to $15 \mathrm{MHz}$; and second, the dielectric properties of extracellular and intracellular media sustained only linear temperature-induced variations during spectrin denaturation, above mentioned dielectric changes were supposed to represent the contribution of native spectrin MS to the dielectric properties of plasma membranes just prior the $T_{\mathrm{A}}$. The frequency analysis of above mentioned dielectric changes, based on the traditional methods of dielectric spectroscopy, has revealed two dielectric relaxations associated to this contribution (Figs. 5A and 8A).

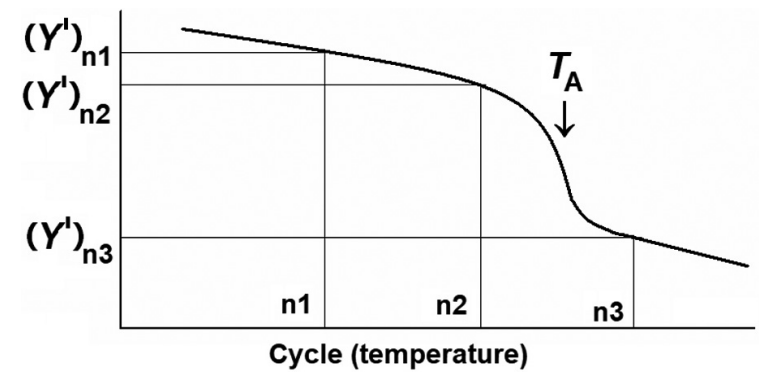

Figure 4. Schematic view of the temperature dependence of $Y$ ' of human RBC suspension at $T_{A}$ (denaturation temperature of RBC spectrin) indicated by arrow. $\mathrm{n} 1, \mathrm{n} 2$ and $\mathrm{n} 3$ are the numbers of cycles (numbers of sweeps on frequency) for data collection, corresponding to different temperatures around $T_{A}$. The hematocrit, frequency and heating rate were $0.55,1 \mathrm{MHz}$ and $1.5^{\circ} \mathrm{C} / \mathrm{min}$, respectively. 
A

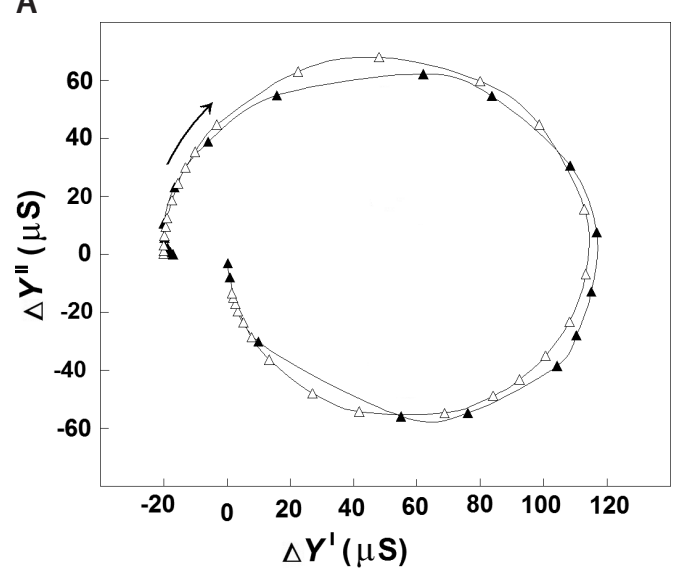

B

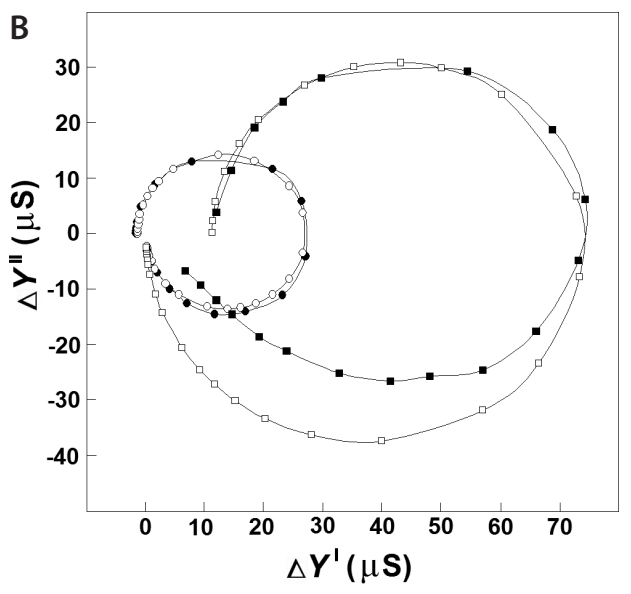

Figure 5. A. Plot of the complex admittance changes at $T_{\mathrm{A}}$ for suspensions containing either human RBCs or their resealed ghost membranes $(\boldsymbol{\Delta})$. The changes in imaginary admittance, $\Delta Y^{\prime \prime}$ $(\mu S)$ are plotted against the changes in real admittance $\Delta Y^{\prime}(\mu S)$ of suspensions. Curved arrow indicates the increase in frequency from $20 \mathrm{kHz}$ to $15 \mathrm{MHz}$. The open triangles $(\Delta)$ indicate the

model plot, $y^{\prime \prime} v s$. y', for these relaxations, obtained as explained for Fig. 6. (Other details as for Fig. 4.) B. Plots of complex admittance changes at $T_{\mathrm{A}}$ for suspensions containing bovine $\operatorname{RBCs}(\bullet, O)$ and chicken $\operatorname{RBCs}(\boldsymbol{\square}, \square)$. The full symbols correspond to experimental data, while open symbols indicate the model plot, respectively. The changes in complex admittance, $\Delta Y^{\prime \prime}(\mu S)$ are plotted against the changes in real admittance $\Delta Y^{\prime}(\mu \mathrm{S})$ of suspensions. (Other details as for Fig. 5A.)

\section{Dielectric relaxations on spectrin MS of human $R B C s$}

Figure 5A (full symbols) shows for the first time the admittance change plot, $\Delta Y^{\prime \prime} v s . \Delta Y^{\prime}$, obtained with human RBCs and their resealed ghost membranes. The respective impedance change plot $\left(-\Delta Z^{\prime \prime} v s . \Delta Z^{\prime}\right)$ has been previously reported (Ivanov and Paarvanova 2016, 2019a). Similar to the $-\Delta Z$ " vs. $\Delta Z^{\prime}$ plot, the $\Delta Y^{\prime \prime} v s . \Delta Y^{\prime}$ plot also depicts two semicircle arcs, one placed above and another one below the abscissa (real) axis, revealing the recently published two single-time dielectric relaxations (Ivanov and Paarvanova 2019a) here designated as $\beta_{\text {sp }}$ and $\gamma 1_{\text {sp }}$, respectively. The upper arc expressed the $\beta_{\mathrm{sp}}$ relaxation, whose $f_{\mathrm{c}}$ (further called $f_{\beta \mathrm{sp}}$ ) coincided with the $f_{\mathrm{c}}$ of the interfacial $\beta$ dispersion on the lipid-protein membrane (further called $f_{\beta}$ ). The lower arc revealed the $\gamma 1_{\text {sp }}$ relaxation whose $f_{c}$ (further called $f_{\gamma 1 \text { sp }}$ ) has
A

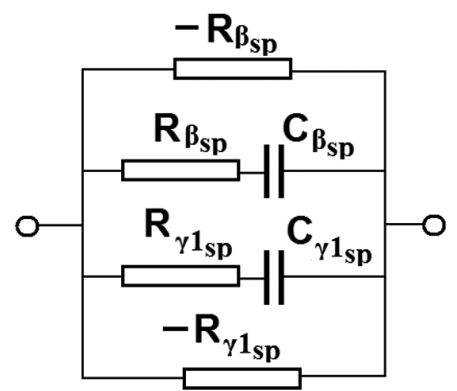

C

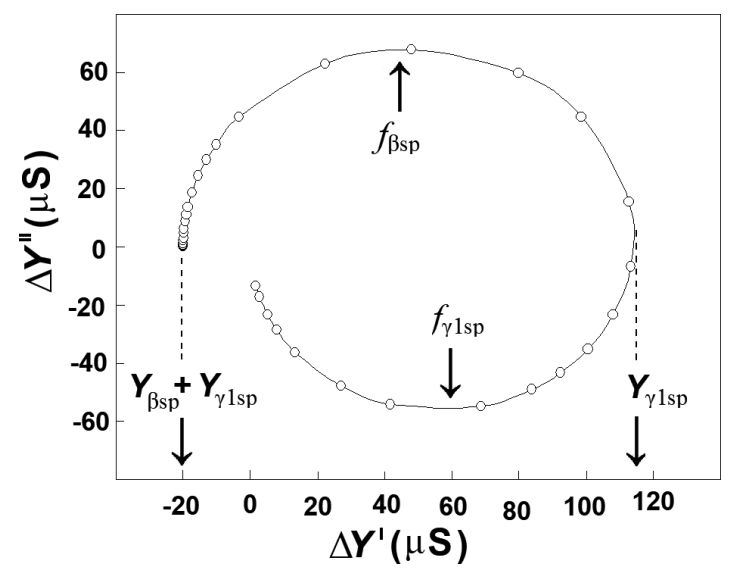

B

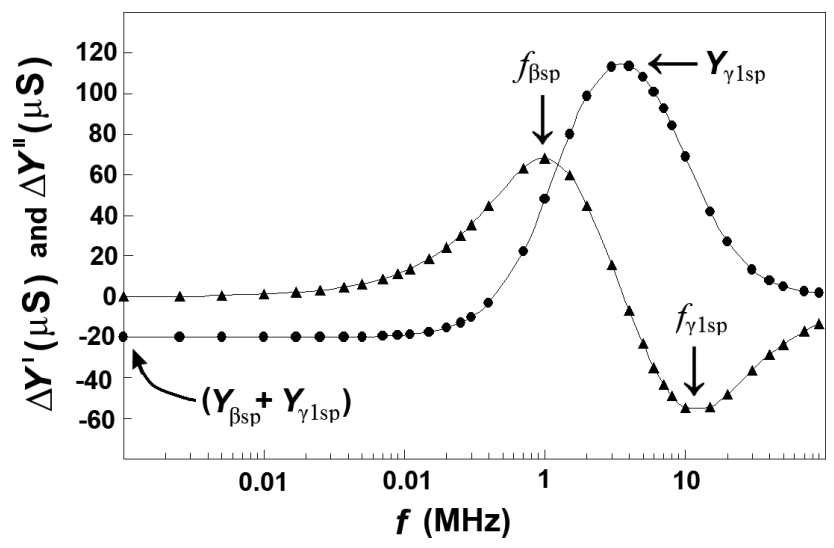

Figure 6. A. Equivalent electric circuit, modeling the experimental admittance data for the $\beta_{\mathrm{sp}}$ and $\gamma_{1 \mathrm{sp}}$ dielectric relaxations on the spectrin of MS of human RBCs and RBC ghost membranes. Using the frequency dependencies of $\Delta Y^{\prime}(\mu \mathrm{S})(\bullet)$ and $\Delta Y^{\prime \prime}(\mu \mathrm{S})(\mathbf{\bullet})$, the values of model admittances, $Y_{\beta s p}$ and $Y_{\gamma 1 s p}$, of model resistances, $R_{\beta \mathrm{sp}}=1 / Y_{\beta \mathrm{sp}}$ and $R_{\gamma 1 \mathrm{sp}}=1 / Y_{\gamma 1 \mathrm{sp}}$, and of the characteristic frequencies, $f_{\beta s p}$ and $f_{\gamma 1 s p}$, of the $\beta_{\text {sp }}$ and $\gamma_{1 \text { sp }}$ relaxations are determined as indicated (B and $\mathbf{C}$ ). The values of $C_{\beta \text { sp }}$ and $C_{\gamma 1 \mathrm{sp}}$, were calculated as explained in the text. 
been shown to depend on the viscosity and ionic concentration of cytosol (Ivanov and Paarvanova 2016). According to above publications the negative direction of the second arc is due to the cytosolic disposition of MS.

\section{Model representation of dielectric relaxations on the MS of human RBCs}

To obtain quantitative description of the $\beta_{\mathrm{sp}}$ and $\gamma 1_{\mathrm{sp}}$ dielectric relaxations on MS we used an electric model adequate to the obtained admittance change plot (Fig. 5A). The main part of the model represented two parallel circuits, each containing a resistor and a capacitor, connected in series (Fig. 6A). The model parameters, $R_{\beta \text { sp }}$ and $C_{\beta \text { sp }}$, of the upper circuit represented the best fit values for the $\beta_{\mathrm{sp}}$ relaxation, while the $R_{\gamma 1 \mathrm{sp}}$ and $C_{\gamma 1 \text { sp }}$ of the lower circuit were the best fit values for the $\gamma 1_{\text {sp }}$ relaxation.

For a series combination of resistance, $R_{\beta s p}$, and capacitance, $C_{\beta \text { sp }}$, the complex impedance is given by $Z_{\beta \text { sp }}{ }^{*}$ $=R_{\beta s p}-\mathrm{i} /\left(\omega C_{\beta \mathrm{sp}}\right)$, where the angular frequency, $\omega=2 \pi f$. Hence, the complex admittance is $Y_{\beta \mathrm{sp}}{ }^{*}=1 / Z_{\beta \mathrm{sp}}{ }^{*}=Y_{\beta \mathrm{sp}}$ ' $+\mathrm{j} Y_{\beta \mathrm{sp}}$ ", where:

$$
\begin{aligned}
& Y_{\beta \mathrm{sp}}{ }^{\prime}=R_{\beta \mathrm{sp}}{ }^{-1} \omega^{2} R_{\beta \mathrm{sp}}{ }^{2} C_{\beta \mathrm{sp}}{ }^{2} /\left(1+\omega^{2} R_{\beta \mathrm{sp}}{ }^{2} C_{\beta \mathrm{sp}}{ }^{2}\right) \\
& Y_{\beta \mathrm{sp}}{ }^{\prime}=\omega C_{\beta \mathrm{sp}} /\left(1+\omega^{2} R_{\beta \mathrm{sp}}{ }^{2} C_{\beta \mathrm{sp}}{ }^{2}\right)
\end{aligned}
$$

The corresponding admittance plot, $Y_{\beta \text { sp }}$ vs. $Y_{\beta \text { sp, is }}$, is a semicircle intersecting the real axis at the origin and at another point $1 / R_{\beta \mathrm{sp}}$. The semicircle could describe a singletime dielectric relaxation whereat the product $R_{\beta \mathrm{sp}} C_{\beta \mathrm{sp}}$ $=1 /\left(2 \pi f_{\beta s p}\right)$ and $f_{\beta s p}$ is the characteristic frequency of the relaxation.

In case another series combination of resistance, $R_{\gamma 1 \mathrm{sp}}$, and capacitance, $C_{\gamma 1 \mathrm{sp}}$, is connected in parallel to the former, its complex admittance ads to that of the former and the resulting complex admittance plot will depict two

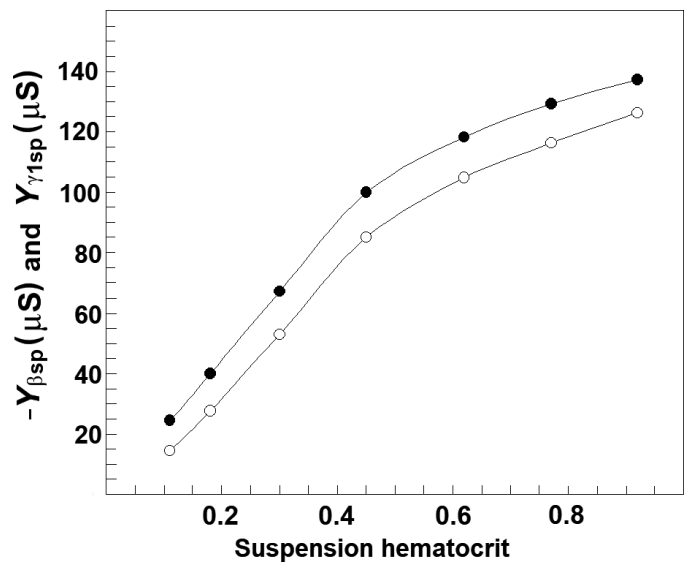

Figure 7. Dependence of model admittances, $Y_{\beta \mathrm{sp}}$ and $Y_{\gamma 1 \mathrm{sp}}$, for the $\beta_{\text {sp }}$ and $\gamma_{1 s p}$ dielectric relaxations on the spectrin of MS of human RBCs and RBC ghost membranes on the suspension hematocrit. $-Y_{\beta s p}(\mu \mathrm{S})$ and $Y_{\gamma 1 \mathrm{sp}}(\mu \mathrm{S})$ are expressed as dependent on the cell volume fraction. (Other details as for Fig. 5A.)

semicircles. At low and high frequencies, however, the real component of the resultant complex admittance tends to zero and to the conductance of the two resistances, $R_{\beta \text { sp }}$ and $R_{\gamma 1 \text { sp }}$, connected in parallel, correspondingly. By contrast, $\Delta Y^{\prime}$ demonstrated just the opposite behaviour tending to zero at high frequencies and to the conductance of the two resistances, $R_{\beta \text { sp }}$ and $R_{\gamma 1 \text { sp }}$, connected in parallel, at low frequencies (Fig. 6B). To eliminate this inconsistency, we added two resistors having negative resistance values, $-R_{\beta \mathrm{sp}}$ and $-R_{\gamma 1 \mathrm{sp}}$, connected in parallel to the main part, as shown in Fig. 6A. The usage of resistors with negative resistance values is justified because the admittance change plot, $\Delta Y^{\prime \prime} v s$. $\Delta Y^{\prime}$, is based on the differences between various admittance values, and the result could be both positive and negative one. Thus, the total complex admittance, $y^{*}=y^{\prime}+\mathrm{j} y^{\prime \prime}$, of the model circuit obtains the final form:
A

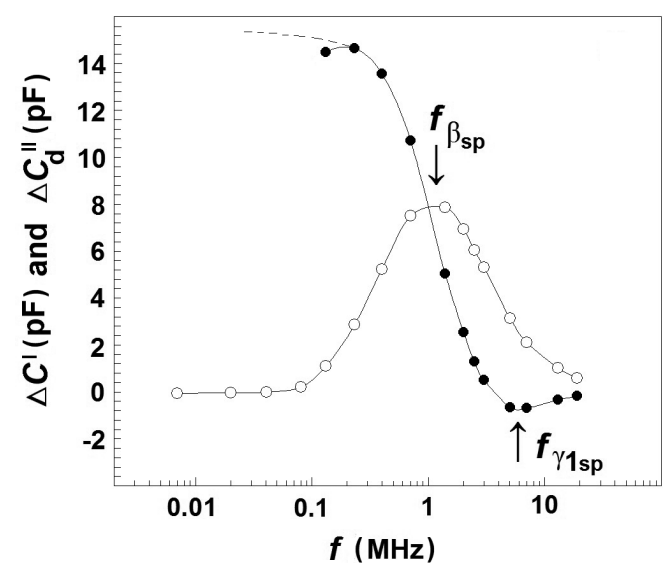

B

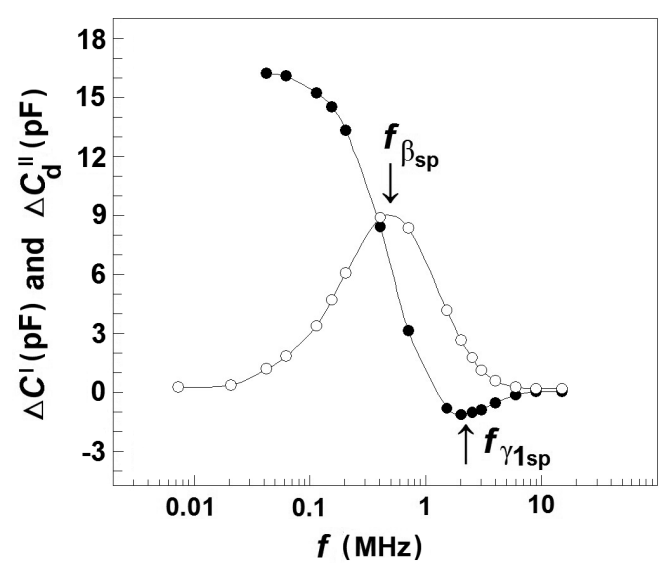

Figure 8. Frequency dependences of the changes at $T_{\mathrm{A}}$ in the real capacitance, $\Delta C^{\prime}(\bullet)$, and in the differential dielectric loss, $\Delta C_{\mathrm{d}}$ " $(\mathrm{O})$, of suspensions containing either human $\mathrm{RBCs}$ or their resealed ghost membranes (A) and chicken RBCs (B). Arrows indicate the characteristic frequencies, $f_{\beta \text { sp }}$ and $f_{\gamma 1 \mathrm{sp}}$, of the $\beta_{\mathrm{sp}}$ and $\gamma_{1 \text { sp }}$ dielectric relaxations on spectrin MS. (Other details as for Fig. 3 and 5.) 


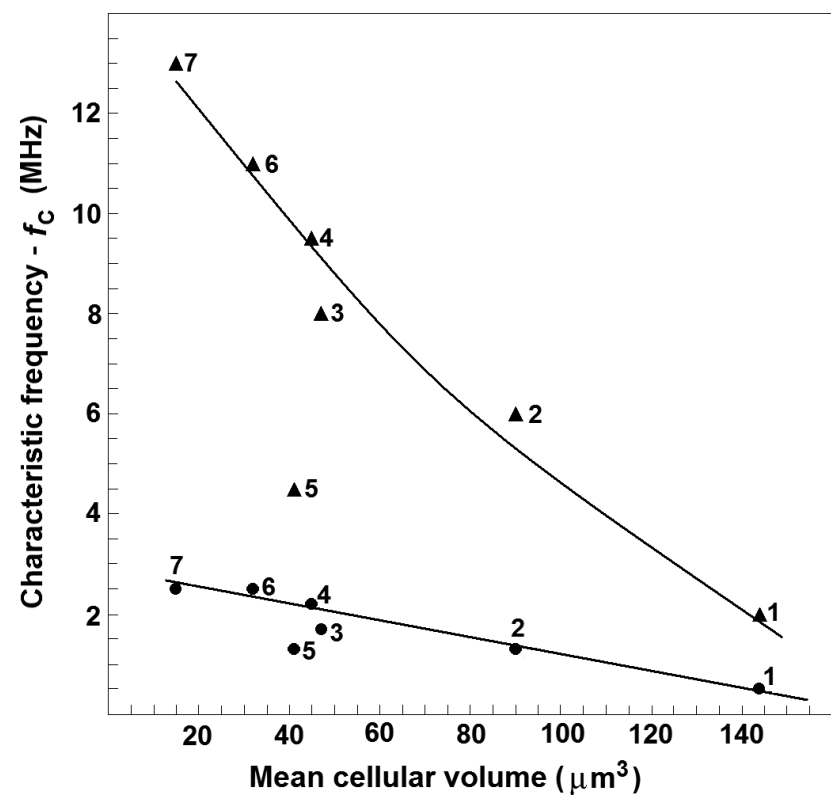

Figure 9. Effect of the mean volume of RBC species on the characteristic frequencies of $\beta_{\mathrm{sp}}(\bullet)$ and $\gamma_{1 \mathrm{sp}}(\boldsymbol{\Delta})$ dielectric relaxations on the MS spectrin. The $f_{\mathrm{c}}(\mathrm{MHz})$ is ploted against the mean cellular volume MCV $\left(\mu^{3}\right)$. Numbers indicate the RBC species: 1, "unstrained" chicken; 2 , human; 3 , rat; 4 , bovine; 5 , horse; 6 , sheep; 7 , goat.

$y^{\prime}=R_{\beta \mathrm{sp}}{ }^{-1} \omega^{2} R_{\beta \mathrm{sp}}{ }^{2} C_{\beta \mathrm{sp}}{ }^{2} /\left(1+\omega^{2} R_{\beta \mathrm{sp}}{ }^{2} C_{\beta \mathrm{sp}}{ }^{2}\right)+$ $+R_{\gamma 1 \mathrm{sp}}{ }^{-1} \omega^{2} R_{\gamma 1 \mathrm{sp}} C_{\gamma 1 \mathrm{sp}} /\left(1+\omega^{2} R_{\gamma 1 \mathrm{sp}}{ }^{2} C_{\gamma 1 \mathrm{sp}}{ }^{2}\right)-1 / R_{\beta \mathrm{sp}}-1 / R_{\gamma 1 \mathrm{sp}}$ $y^{\prime \prime}=\omega C_{\beta \mathrm{sp}} /\left(1+\omega^{2} R_{\beta \mathrm{sp}}{ }^{2} C_{\beta \mathrm{sp}}{ }^{2}\right)+\omega C_{\gamma 1 \mathrm{sp}} /\left(1+\omega^{2} R_{\gamma 1 \mathrm{sp}}{ }^{2} C_{\gamma 1 \mathrm{sp}}{ }^{2}\right)$

The best fit values of $R_{\beta \mathrm{sp}}, C_{\beta \mathrm{sp}}, R_{\gamma 1 \mathrm{sp}}$ and $C_{\gamma 1 \mathrm{sp}}$, were found by iteration as previously explained (Ivanov and Paarvanova 2019a). Briefly, the values of the characteristic frequencies, $f_{\beta \text { sp }}$ and $f_{\gamma 1 \mathrm{sp}}$, and the initial values of $R_{\beta \mathrm{sp}}=$ $1 / Y_{\beta \mathrm{sp}}$ and $R_{\gamma 1 \mathrm{sp}}=1 / Y_{\gamma 1 \mathrm{sp}}$ were determined by the ex- perimental frequency curves of $\Delta Y^{\prime}$ and $\Delta Y^{\prime \prime}$ as indicated in Fig. 6B. Hence, the initial values of $C_{\beta \text { sp }}$ and $C_{\gamma 1 \mathrm{sp}}$ were calculated by the products $R_{\beta s p} C_{\beta s p}=1 /\left(2 \pi f_{\beta s p}\right)$ and $R_{\gamma 1 \mathrm{sp}} C_{\gamma 1 \mathrm{sp}}=1 /\left(2 \pi f_{\gamma 1 \mathrm{sp}}\right)$. During the next several iterations the values of $Y_{\beta \text { sp }}$ and $Y_{\gamma 1 \text { sp }}$ only were adjusted until the obtained model plot, $y$ " vs. $y$,' (Fig. 6C) coincided with the experimental $\Delta Y^{\prime \prime}$ vs. $\Delta Y^{\prime}$ plot as indicated in Fig. $5 \mathrm{~A}$.

Next, the dependences of model admittances, $Y_{\beta s p}=1 /$ $R_{\beta s p}$ and $Y_{\gamma 1 \mathrm{sp}}=1 / R_{\gamma 1 \mathrm{sp}}$, on the RBC suspension hematocrit were determined. These dependences were linear up to the hematocrit values of 0.55 (Fig. 7), while they tended to saturation at denser suspensions. The $C_{\beta \text { sp }}$ and $C_{\gamma 1 \text { sp }}$ depended likewise on the RBC suspension hematocrit (not shown).

Based on the data in Fig. 7 and similar data for $C_{\beta \text { sp }}$ and $C_{\gamma 1 \text { sp }}$, it could be assumed that $Y_{\beta \text { sp }}$ and $Y_{\gamma 1 \text { sp }}$, on the one hand, and $C_{\beta s p}$ and $C_{\gamma 1 \text { sp }}$, on the other, reflected the energy loss and charge accumulation during the $\beta_{\text {sp }}$ and $\gamma_{1 \mathrm{sp}}$ relaxations in suspended RBCs, respectively. Hence, it was convenient to use their ratios. Such are the energy dissipation ratio, $-Y_{\gamma 1 \mathrm{sp}} / Y_{\beta \mathrm{sp}}$, and energy storage ratio, $-C_{\gamma 1 \mathrm{sp}} / C_{\beta \mathrm{sp}}$, which represent the amount of energy, dissipated and stored, respectively, on spectrin during the $\gamma 1_{\text {sp }}$ relaxation relative to that in $\beta_{\mathrm{sp}}$ relaxation. For human RBCs, suspended in working medium, the values of these parameters are shown in Table 1 and the obtained characteristic frequencies, $f_{\beta s p}$ and $f_{\gamma 1 \mathrm{sp}}$, are shown in Table 2 .

The conductance of suspension medium (especially at $\mathrm{NaCl}$ concentrations above $20 \mathrm{mM}$ ) short-circuits the impedance of suspended RBCs. Due to the Ohm's law; it reduces the assayed changes in the impedance of heated suspension at $T_{\mathrm{A}}$, produced by the changes in the complex impedance of RBCs. In this study the admittance change plot was preferred to the impedance change plot because it appeared not affected by the conductance ( $\mathrm{NaCl}$ concentration) of suspension medium, at least between 10 and $50 \mathrm{mM}$ $\mathrm{NaCl}$ (not shown). This outcome arises from the temperature correction of admittance changes at $T_{\mathrm{A}}$. Due to the Ohm's

Table 1. Model study of the $\beta_{\mathrm{sp}}$ and $\gamma_{1 \mathrm{sp}}$ dielectric relaxations on the spectrin-based membrane skeleton of human and mammal RBCs and "unstrained" RBCs of chicken

\begin{tabular}{lrcccccccrr}
\hline Species & $n$ & $Y_{\beta s p}(\mu \mathrm{S})$ & $C_{\beta \mathrm{sp}}(\mathrm{pF})$ & $Y_{\gamma 1 \mathrm{sp}}(\mu \mathrm{S})$ & $C_{\gamma 1 \mathrm{sp}}(\mathrm{pF})$ & $-Y_{\gamma 1 \mathrm{sp}} / Y_{\beta \mathrm{sp}}$ & $-C_{\gamma 1 \mathrm{sp}} / C_{\beta \mathrm{sp}}$ & $C^{\prime}(\mathrm{pF})$ & $\Phi(\mu \mathrm{m})$ & $\mathrm{MCV}\left(\mu \mathrm{m}^{3}\right)$ \\
\hline Chicken & 5 & -57.6 & -20.2 & 63.7 & 4.46 & 1.106 & 0.18 & 60 & $12.1 / 7.3$ & 144 \\
Human & 10 & -130.0 & -18.8 & 112 & 2.38 & 0.861 & 0.13 & 45 & 7.4 & 90 \\
Rat & 8 & -88.5 & -17.6 & 76.0 & 1.73 & 0.859 & 0.13 & 36 & 6 & 47 \\
Horse & 1 & -51.0 & -4.51 & 41.0 & 0.69 & 0.804 & 0.24 & 24 & 5.7 & 45 \\
Bovine & 2 & -37.4 & -6.62 & 36.0 & 0.88 & 0.963 & 0.14 & 25 & 5.5 & 41 \\
Sheep & 2 & -56.0 & -4.46 & 50.0 & 0.66 & 0.893 & 0.21 & 20 & 4.5 & 32 \\
Goat & 2 & -27.4 & -1.8 & 18.2 & 0.17 & 0.664 & 0.21 & 16 & 3.5 & 15 \\
\hline
\end{tabular}

The RBCs were suspended in working medium at hematocrit 0.55 . Given are only the mean values, the deviations were less than $10 \%$ of the mean value. Other details are explained in Results section. Adduced are the mean diameter $(\Phi)$ and mean cellular volume (MCV) of RBCs adopted from Windberger et al. (2003). C', static capacitance of suspension; $n$, number of tried individuals from each species. 
Table 2. Characteristic frequency values of $\beta_{\mathrm{sp}}$ and $\gamma 1_{\mathrm{sp}}$ dielectric relaxations on the MS spectrin of human and mammal RBCs and "unstrained" RBCs of chicken

\begin{tabular}{|c|c|c|c|c|c|c|c|}
\hline \multirow[b]{2}{*}{ Species } & \multirow{2}{*}{$n$} & \multirow{2}{*}{$\frac{f_{\beta}(\mathrm{MHz})}{C^{\prime} \text { vs. } f}$} & \multicolumn{3}{|c|}{$f_{\beta s p}(\mathrm{MHz})$} & \multicolumn{2}{|c|}{$f_{\gamma 1 s p}(\mathrm{MHz})$} \\
\hline & & & $\Delta C^{\prime}$ vs. $f$ & $\Delta C^{\prime \prime}$ vs. $f$ & $\Delta Y^{\prime \prime}$ vs. $f$ & $\Delta C^{\prime} v s . \mathrm{f}$ & $\Delta Y^{\prime \prime}$ vs. $f$ \\
\hline Chicken & 5 & 1.0 & 0.6 & 0.5 & 0.6 & 2.0 & 3.0 \\
\hline Human & 10 & 1.6 & 1.3 & 1.3 & 1.3 & 7.0 & 7.0 \\
\hline Rat & 8 & 0.7 & 0.8 & 0.8 & 0.8 & 8.0 & 8.0 \\
\hline Horse & 1 & 2.2 & 1.8 & 2.2 & 1.8 & 9.5 & 9.5 \\
\hline Bovine & 2 & 2.3 & 1.3 & 1.3 & 1.2 & 4.5 & 5.5 \\
\hline Sheep & 2 & 3.3 & 2.7 & 2.5 & 2.0 & 11.0 & 10 \\
\hline Goat & 2 & 4.5 & 2.5 & 2.5 & 2.0 & 13.0 & 12 \\
\hline
\end{tabular}

The values were determined from the indicated frequency dependencies. $f_{\beta}$, frequency of the $\beta$ relaxation on the lipid bilayer; $f_{\beta s p}$, frequency of the $\beta_{\text {sp }}$ relaxation on spectrin MS; $f_{\gamma 1 s p}$, frequency of the $\gamma 1_{\text {sp }}$ relaxation on spectrin MS. (Other details as for Table 1.)

law, this correction annihilates the accompanying changes in admittances of all paths of the current outside the RBCs as they are parallel to the paths through the RBCs. Hence, in contrast to the impedance change plot, the admittance change plot (Fig. 5A) could be used to obtain the strength of respective relaxations.

\section{Frequency dependencies of $\Delta C^{\prime}$ and $\Delta C_{d}$ " of human $R B C s$}

As previously indicated (Ivanov and Paarvanova 2019a), the frequency dependences of $\Delta C^{\prime}$ and $\Delta C_{\mathrm{d}}$ " for human RBCs (Fig. 8A) revealed the same $\beta_{\mathrm{sp}}$ and $\gamma 1_{\mathrm{sp}}$ relaxations. The values of their characteristic frequencies, $f_{\beta \text { sp }}$ and $f_{\gamma 1 \text { sp }}$, are indicated by arrows and shown in Table 2 . The $f_{\beta \text { sp }}$ and $f_{\gamma 1 \text { sp }}$ had the same values when determined using either complex admittance or complex capacitance change plots. In case the $f_{\beta s p}$ and $f_{\gamma 1 \text { sp }}$ were determined using the complex impedance change plot their values were substantially decreased due to the reciprocal dependence of complex impedance on complex admittance.

\section{Dielectric relaxations on the spectrin MS of RBCs from mammal species}

Plots of complex admittance changes at $T_{\mathrm{A}}$, similar to that of human RBCs (Fig. 5A), were obtained with rat, horse, bovine, sheep and goat RBCs. For bovine RBCs this plot is shown in Fig. 5B. In addition, frequency dependences of $\Delta C^{\prime}$ and $\Delta C_{\mathrm{d}}$ ", as those obtained with human RBCs (Fig. $8 \mathrm{~A}$ ) were obtained with rat, horse, bovine, sheep and goat RBCs (not shown). The best fit values of $Y_{\beta \text { sp }}, C_{\beta \text { sp }}, Y_{\gamma 1 \text { sp }}$ and $C_{\gamma 1 \text { sp }}$ for these RBCs, obtained by above mentioned model study, are also shown in Table 1 . The characteristic frequencies, $f_{\beta \text { sp }}$ and $f_{\gamma 1 \text { sp }}$, obtained by the $\Delta Y^{\prime \prime}$ vs. $\Delta Y^{\prime}$ plots and by the frequency dependences of $\Delta C^{\prime}$ and $\Delta C_{\mathrm{d}}{ }^{\prime \prime}$ are shown in Table 2.

\section{Dielectric relaxations on the MS of chicken $R B C s$}

With human RBCs and their ghost membranes, as well as with the other enucleated RBCs of mammals, the admittance change plots (Fig. 5A) and the frequency dependences of $\Delta C^{\prime}$ and $\Delta C_{\mathrm{d}}$ " (Fig. $8 \mathrm{~A}$ ) were readily obtainable without the need of special conditions and reagents. For example, the cells could be freshly drawn or incubated at $4^{\circ} \mathrm{C}$ overnight, or kept at room temperature for up to 6 hours. To obtain such plots with chicken RBCs we met a serious problem. With RBCs, isolated from freshly drawn chicken blood, except of the continuous effect of temperature, no threshold changes in the dielectric parameters of suspension were detected up to $60^{\circ} \mathrm{C}$, which is far beyond the $T_{\mathrm{A}}$ of these cells. Such a negative result was also obtained using chicken RBCs, kept in a refrigerator for up to $30 \mathrm{~h}$, and subjected to test heating just after the cold storage. Above negative results could be possibly due to the cytoskeleton of chicken RBCs and its multiple connections with the MS of these cells. According to Miller and Solomon (1984) and Kim et al. (1987), the marginal band of cytoskeleton of chicken RBCs became depolymerized during cold treatment however, the next test heating allowed them to completely re-grow prior to reaching the $T_{\mathrm{A}}$. Possibly, the dielectric activity of MS of chicken RBCs with intact or re-grown cytoskeleton was heavily inhibited that prevented its discrimination in respect to the negligible dielectric activity of denatured spectrin.

To test above explanation, we decided to irreversibly change the state of cytoskeleton of chicken RBCs depolymerizing the marginal band by cold treatment, followed by addition of colchicine $(15 \mathrm{mM})$ or vinblastine $(30 \mu \mathrm{M})$. Nevertheless, no changes at $T_{\mathrm{A}}$ in the dielectric properties of latter cells were noticed in case the duration of cold treatment was up to $3 \mathrm{~h}$, which is sufficient to disassemble the marginal band alone. Prolonging the cold treatment up to $6 \mathrm{~h}$, such changes appeared however, they were still very small. After 12-h 
cold-exposure of cells the changes were already sufficient to obtain reliable plots for the admittance and capacitance changes at $T_{\mathrm{A}}$ (not shown). The strongest dielectric changes at $T_{\mathrm{A}}$ were obtained in so called "unstrained" RBCs, exposed to the cold for about $20-30 \mathrm{~h}$, as demonstrated in Figs. $5 \mathrm{~B}$ and $8 \mathrm{~B}$. The latter changes revealed $\beta_{\mathrm{sp}}$ and $\gamma 1_{\mathrm{sp}}$ relaxations whose characteristic frequencies are shown in Table 2 . The absence of colchicine and vinblastine, even at such longterm cold treatment, prevented the appearance of dielectric changes at $T_{\mathrm{A}}$. These results indicate that i) neither metabolic depletion, nor the short-term irreversible disassembly of marginal band alone could induce such changes and, ii) the disassembly of marginal band, achieved by exposure of cells to $4^{\circ} \mathrm{C}$ for approximately one hour, was insufficient premise as there should be additional cold exposure (approximately $20 \mathrm{~h}$ at $4^{\circ} \mathrm{C}$ ) in order to eliminate the immobilization of MS as judged by the appeared dielectric activity. The extra cold treatment was possibly needed to disrupt another cytoskeleton component, in addition to the marginal band.

In another experiments, packed chicken RBCs were incubated at $4^{\circ} \mathrm{C}$ for $30 \mathrm{~h}$ and suspended in cold $\left(4^{\circ} \mathrm{C}\right)$ isotonic medium of mannit and $30 \mathrm{mM}$ phosphate buffer, $\mathrm{pH} 7.4$ for $2 \mathrm{~h}$. During the next test heating, these cells demonstrated dielectric changes at $T_{\mathrm{A}}$ similar to those presented in Figs. $5 \mathrm{~B}$ and $8 \mathrm{~B}$. Thus, using $30 \mathrm{mM}$ phosphate buffer instead of colchicine or vinblastine, practically the same mobilization effect on the dielectric activity of MS of chicken RBCs was obtained. This outcome could be explained with the ability of phosphate anions to increase the dynamic instability of tubulin, isolated from chicken RBCs (Trinczek et al. 1993). In these experiments the phosphate anions entered the cytosol of chicken RBCs and, according to above report, they possibly impeded the re-growth of marginal band and the related additional portion of cytoskeleton. This assumption was supported by the finding that addition of colchicine (final concentration $15 \mathrm{mM}$ ) together with the phosphate buffer did not change the result obtained with phosphate buffer alone.

The specific flat elongated shape of chicken RBCs (Fig. 2A) was preserved after the irreversible disassembly of their marginal band and even after the extra cold treatment resulting in "unstraining" their MS (Fig. 2C). According to (Glomski and Pica 2011) the cold-induced disruption of marginal band of chicken RBCs eliminates their elastic resilience to mechanical forces and makes the cells plastic. Nevertheless, the consequent thermal denaturation of MS spectrin did not result in marked deviation of chicken RBC shape from the native one (Fig. 2D) although mechanical properties of cells could further aggravate.

\section{Static capacitance and $f_{c}$ of $\beta$ relaxation of $R B C$ suspensions}

For the needs of our study, we obtained the frequency dependences of real capacitance, $C^{\prime}$, of suspensions contain- ing human and mammal RBCs and "unstrained" chicken RBCs (not shown). To avoid electrode polarization, static capacitance of each RBC suspension was determined by extrapolating the maximal values, forming plateau, to zero frequency. For each species, the $f_{c}$ of $\beta$ relaxation, $f_{\beta}$, on the lipid-protein membrane was determined at the inflexion point on the frequency dependence of $C$. The obtained values for the static capacitances and for the $f_{\beta}$ are shown in Table 2.

\section{Discussion}

Nevertheless, that $Y_{\gamma 1 \text { sp }}$ and $Y_{\beta s p}$ both varied about 5 times for the group of seven studied RBC species, their ratio changed slightly, $-Y_{\gamma 1 \mathrm{sp}} / Y_{\beta \text { sp }}=0.879 \pm 0.126$ (Table 1). This shows that spectrin of each RBC species irreversibly dissipated almost equal amounts of energy during both relaxations. In our previous studies on human RBCs, the $-Y_{\gamma 1 \mathrm{sp}}$ $/ Y_{\beta \text { sp }}$ ratio has been shown to change on separation of MS from the lipid membrane (Ivanov and Paarvanova 2019a) and in the presence of polar solvents (Ivanov and Paarvanova 2019b). Compared to control human RBCs, it decreased on specific severing the spectrin-band 3 attachment site and in the presence of aprotic solvents while it increased on disturbing the spectrin-actin interaction and in the presence of protic solvents. Above findings indicate the importance of this parameter for the evaluation of MS detachment.

The energy storage ratio, $-C_{\gamma 1 \mathrm{sp}} / C_{\beta \mathrm{sp}}=0.135 \pm 0.040$, also did not differ vastly from species to species (Table 1 ). However, the low value of latter ratio showed asymmetric ability of spectrin to generate charges during the two relaxations. It indicates that, compared to $\gamma 1_{\text {sp }}$ relaxation, about seven times more charges were generated on spectrin during the $\beta_{\text {sp }}$ relaxation. This finding is in line with the previous assertion that $\beta_{\text {sp }}$ relaxation reflects piezo effect on spectrin, which effect is especially efficient in charge generation.

Above results assume that complex admittance change plot, $\Delta Y^{\prime \prime} v s$. $\Delta Y$, expressed the irreversible loss of energy on MS during the $\beta_{\mathrm{sp}}$ and $\gamma 1_{\mathrm{sp}}$ relaxations. As the two relaxations dissipated almost equal amounts of energy on MS they were represented by two semicircles with almost equal areas (Fig. 5A and B). On the other hand, capacitance change plot, $\Delta C_{\mathrm{d}}$ " $v$ s. $\Delta C^{\prime}$ (Ivanov and Paarvanova 2019a), could be assumed expressing the reversible accumulation of charges, respectively electric energy, on MS during the $\beta_{\mathrm{sp}}$ and $\gamma 1_{\mathrm{sp}}$ relaxations. As the $\gamma 1_{\text {sp }}$ relaxation accumulated about seven times smaller amount of charges, compared to $\beta_{\text {sp }}$ relaxation, it was expressed by a small (almost indistinguishable) semicircle at the high frequency end of the big semicircle of $\beta_{\text {sp }}$ relaxation (Ivanov and Paarvanova 2019a) although it could be detected by the slight negative peak on the high end of the frequency plot of $\Delta C^{\prime}$ (Fig. $8 \mathrm{~A}$ and $\mathrm{B}$ ). 
Result, similar to that shown in Fig. 7, has been reported about the shape of complex relative permittivity plot revealing the beta relaxation in RBC suspensions (Hanai et al. 1979). At hematocrit values $<0.60$ this plot had the shape of a semicircle, while with denser suspensions it became increasingly distorted especially at the low frequency end. However, the real part of dielectric permittivity has been shown more sensitive to the hematocrit of RBC suspensions and intercellular interactions (Lisin et al. 1996). Up to the hematocrit values of 0.20 it depended linearly while at greater hematocrit values the linear dependence was disturbed. Apparently, the magnitude of dielectric changes, induced by the heat denaturation of under-membrane skeleton at $T_{\mathrm{A}}$, was less sensitive to the intercellular interactions.

For spherical cells, suspended at a given cytocrit value, the strength of $\beta$ relaxation and the time for charge accumulation on their lipid-protein membrane both increase linearly with the mean radius of cells (Prodan et al. 2008; Glomski and Pica 2011). RBC species, used in this study were not spherical ones and their shape could have specific impact on these dependences (Hayashi et al. 2008). Nevertheless, in general, above dependences could explain the good correlation between the volume of studied RBC species, on one hand, and the static capacitance and the $f_{c}$ of the $\beta$ relaxation of their suspensions, on the other (Table 1 and 2).

As shown in Table 1 and Figure 9, the $f_{\mathrm{c}}$ of $\beta_{\mathrm{sp}}$ and $\gamma 1_{\mathrm{sp}}$ relaxations also displayed strong species variations being lowest in RBC species with highest volume ("unstrained" chicken RBCs) and highest in RBC species with smallest volume (sheep and goat RBCs). Figure 9 exhibits that, with the exception of bovine RBCs, the two characteristic frequencies decreased almost linearly, although at different rates, with the mean volume of studied cells. This finding again indicates different mechanisms for dielectric polarization of spectrin in $\beta_{\text {sp }}$ and $\gamma 1_{\text {sp }}$ relaxations. With bovine and "unstrained" chicken RBCs, the $f_{\mathrm{c}}$ of $\gamma 1_{\text {sp }}$ relaxation had markedly low values, which could be possibly related to the great thermal stability of latter RBC species at $T_{\mathrm{A}}$ (Mosior et al. 1990; see Fig. 2).

The dependence of $f_{\mathrm{c}}$ of $\beta_{\mathrm{sp}}$ relaxation on the volume of $\mathrm{RBC}$ species could be explained assuming that the mechanism of this relaxation in mammal and "unstrained" chicken RBCs was similar to that in human RBCs. This assumption is substantiated by the similar structure of the plasma membranes in studied RBC species and by the data in Table 2 demonstrating coincidence of $f_{\mathrm{c}}$ of $\beta_{\mathrm{sp}}$ relaxation with that of $f_{\mathrm{c}}$ of $\beta$ relaxation on the lipid membrane of studied RBCs.

However, the dependence of $f_{\mathrm{c}}$ of $\gamma 1_{\mathrm{sp}}$ relaxation on the volume of RBC species is hard to explain as there are no similar dependences of the composition and structure of either spectrin or any other MS protein on the volume of RBC species. Just the contrary, spectrin and other basic proteins of MS have similar molecular weight and structure in chicken and mammal RBCs (Whitfield et al. 1983; Birkenmeier et al. 1985; Wasenius et al. 1985; Barker 1991).

In general, spectrin is a tetrameric protein that is formed by the head to-head association of two heterodimers (Shotton et al. 1979). Each heterodimer is composed of one alpha $(280 \mathrm{kDa})$ and one beta $(246 \mathrm{kDa})$ polypeptides wrapped around each other in an anti-parallel fashion forming a righthanded double-helix (McGough and Josephs 1990). The alpha and beta polypeptides are further divided into about 23 (for the alpha-spectrin monomer) and about 17 (for the beta-spectrin monomer) independently folded repeat units. Although the repeat units do not exhibit strict sequence homology, each unit has about 106 residues $(\mathrm{Mr} 12,000)$ and is folded into three alpha-helices arranged in an antiparallel coiled coil (Speicher and Marchesi 1984; Speicher 1986). Peptide bonds have substantial dipole moments that are transmitted to the ends of a helices. Due to the anti-parallel wrapping of its alpha and beta polypeptides the entire spectrin dimer should not have net dipole moment. However, due to the triple spiral structure of repeat units, each repeat unit has been assumed to possess a net dipole moment and to vibrate with the natural frequency of the free vibrations of MS segments (Ivanov and Paarvanova 2016). Provided the electric field penetrated the cytosol of RBC it should align these dipoles and lose energy in case its frequency coincides with the natural frequency of their free vibrations. The above conception for the origin of $\gamma 1_{\text {sp }}$ relaxation has the potential to explain the inverse proportionality of its $f_{\mathrm{c}}$ on the volume of RBC species, based on following considerations.

In a medium of $50 \mathrm{mM} \mathrm{NaCl}$ with $\mathrm{pH} 7.6$ the net charge per spectrin dimer is -50 (Elgsaeter et al. 1976). This huge net negative charge generates electrostatic repulsive force that expands the MS of erythrocytes. The elasticity of tetramer counteracts the repulsive force resulting in a resting size of $\mathrm{MS}$ at the balance point. $\mathrm{NaCl}$ ions partially screen the negative charges and decrease the electrostatic repulsive force shifting the balance point towards smaller size of MS. For example, isolated spectrin dimers shorten by about 3-fold in physiological buffers relative to their size in low ionic strength buffers (DeSilva et al. 1997). Isolated MSs also expand and shrink reversibly in response to variations in ionic strength (Lange et al. 982) whereat the diameter and packed volume of MSs, isolated by Triton-X-100, lineary decreased as the concentration of $\mathrm{NaCl}$ increased from 10 to $150 \mathrm{mM}$ (Elgsaeter et al. 1976).

Recently we studied the effect of above mentioned $\mathrm{NaCl}$ induced elongation-shortening of spectrin tetramers of MS on the $f_{\mathrm{c}}$ of $\gamma 1_{\mathrm{sp}}$ relaxation (Ivanov and Paarvanova 2016; Ivanov et al. 2017). For this purpose, human RBC ghost membranes and their MSs, extracted by Triton-X-100, were used. Increasing $\mathrm{NaCl}$ concentration of MS milieu from 20 to $150 \mathrm{mM}$, the $f_{\mathrm{c}}$ of $\gamma 1_{\mathrm{sp}}$ relaxation (measured on the impedance change plot which lowers the values compared to the 
Table 3. Effect of the osmotic change in volume on the "effective" surface area of human RBCs and on the $f_{\mathrm{c}}$ of $\gamma 1_{\mathrm{sp}}$ relaxation, $f_{\gamma 1 \mathrm{sp}}$, of their MS (measured on the impedance change plot)

\begin{tabular}{lccc}
\hline Osmolarity $(\mathrm{mOsm})$ & $f_{\gamma 1 \mathrm{sp}}(\mathrm{MHz})$ & Change in volume & Change in "effective" surface area \\
\hline 200 & 2.5 & Pre-lytic swelling & No change \\
300 (isotonic) & 2.5 & No change & No change \\
600 & 5.0 & Shrinkage & Decrease \\
750 & 8.0 & Shrinkage & Decrease \\
900 & 10 & Shrinkage & Decrease \\
\hline
\end{tabular}

The hematocrit was 0.55 and the suspension media contained $10 \mathrm{mM} \mathrm{NaCl}$ and appropriate concentration of mannit to obtain the indicated osmolarity.

admittance and capacitance change plots) linearly increased from $0.8 \mathrm{MHz}$ to 6 and $9 \mathrm{MHz}$, respectively.

In this study, the volume of human RBCs and their surface area were changed suspending the cells in hypertonic and hypotonic media and the effect on the $f_{\mathrm{c}}$ of $\gamma 1_{\text {sp }}$ relaxation measured. For this purpose, we modified the method of (Johnson et al. 1980) suspending the cells in media containing $10 \mathrm{mM} \mathrm{NaCl}$ and proper concentrations of mannit. Compared to the isotonic medium of $300 \mathrm{mOsm}$, the RBCs shrunk when suspended in hypertonic media decreasing the surface area free of spicules ("effective" surface area) and of their MS. At such condensed state of MS, the $f_{\mathrm{c}}$ of $\gamma 1_{\mathrm{sp}}$ relaxation (again measured on the impedance change plot) increased with the decrease in $\mathrm{RBC}$ volume and $\mathrm{RBC}$ "effective" surface area (Table 3). This increase in the $f_{\mathrm{c}}$ of $\gamma 1_{\text {sp }}$ relaxation was reversible upon washing of RBCs and re-suspending them in isotonic medium. The RBCs, suspended in the hypotonic medium of $200 \mathrm{mOsm}$, swelled still without change in their "effective" surface area and in the state of their MS. In this case, the $f_{\mathrm{c}}$ of $\gamma 1_{\mathrm{sp}}$ relaxation was preserved equal to that of cells suspended in isotonic medium (Table 3).

The above two lines of evidence indicate that the shrinkage of MS of human RBCs was accompanied by an increase in the $f_{\mathrm{c}}$ of $\gamma 1_{\mathrm{sp}}$ relaxation. Similarly, we could formally put forwards the hypothesis that compared to the MS of bigger RBC species ("unstrained" chicken and human RBCs), the MS of smaller RBCs species (sheep and goat RBCs) was more compressed and condensed hence, it has higher $f_{\mathrm{c}}$ of $\gamma 1_{\text {sp }}$ relaxation. This compression of MS could have effect on the mechanical properties of RBCs.

\section{Conclusion}

The presented results substantiate the usefulness of the method of differential thermal dielectroscopy in studying the MS of RBCs of human and other species. Such studies are facilitated by the possibility to isolate RBC ghost membranes and their Triton-X-100 shells. In addition, this method proved applicable for bird (chicken) RBCs provided their cytoskeleton was properly manipulated. So far, two dielectric relaxations have been revealed in studied cells; one apparently related to the electrostriction of lipid membrane and another one possibly depending on the contraction of MS. Further studies are needed to specify the relationship between these relaxations and the mechanical properties of RBCs.

Acknowledgement. This study was supported by the project 4/МФ-2019 of the Thracian University, Stara Zagora, Bulgaria. In addition, B. Paarvanova and B. Tacheva were supported by the Bulgarian Ministry of Education and Science under the National Program for Research "Young Scientists and Postdoctoral Students” (CMD № 577 / 17.08.2018).

\section{References}

Anong WA, Franco T, Chu H, Weis TL, Devlin EE, Bodine DM, An X, Mohandas N, Low PS (2009): Adducin forms a bridge between the erythrocyte membrane and its cytoskeleton and regulates membrane cohesion. Blood 114, 1904.912 https://doi.org/10.1182/blood-2009-02-203216

Asami K (2015): Radiofrequency dielectric properties of cell suspensions. In: Dielectric Relaxations in Biological Systems. Physiscal Principles, Methods, and Applications (Ed. V. Raicu and Y. Feldman), pp. 340-362, Oxford University Press https://doi.org/10.1093/acprof:oso/9780199686513.003.0013

Barker RN (1991): Electrophoretic analysis of erythrocyte membrane proteins and glycoproteins from different species. Comparative Haematology International 1, 155-160 https://doi.org/10.1007/BF00515663

Birkenmeier CS, Bodine DM, Repasky EA, Helfman DM, Hughes SH, Barker JE (1985): Remarkable homology among the internal repeats of erythroid and nonerythroid spectrin. Proc. Natl. Acad. Sci. USA 82, 5671-5675

https://doi.org/10.1073/pnas.82.17.5671

Brandts JF, Erickson L, Lysko K, Schwartz AT, Taverna RD (1977): Calorimetric studies of the structural transitions of the human erythrocyte membrane. The involvement of spectrin in the A transition. Biochemistry 16, 3450-3454 https://doi.org/10.1021/bi00634a024 
Clark AG, Dierkes K, Paluch EK (2013): Monitoring actin cortex thickness in live cells. Biophys. J. 105, 570-580 https://doi.org/10.1016/j.bpj.2013.05.057

DeSilva TM, Harper SL, Kotula L, Hensley P, Curtis PJ, Otvos Jr L, Speicher DW (1997): Physical properties of a single-motif erythrocyte spectrin peptide: a highly stable independently folding unit. Biochemistry 36, 3991-3997 https://doi.org/10.1021/bi962412j

Elgsaeter A, Shotton DM, Branton D (1976): Intramembrane particle aggregation in erythrocyte ghosts. II. The influence of spectrin aggregation. Biochim. Biophys. Acta 426, 101-122 https://doi.org/10.1016/0005-2736(76)90433-8

Eskelinen S, Coakley WT, Tilley D (1985): Thermal denaturation of the erythrocyte cytoskeleton alters the morphological changes associated with osmotic swelling. J. Therm. Biol. 10, $187-190$ https://doi.org/10.1016/0306-4565(85)90038-5

Fung LWM, Kalaw BO, Hatfield RM, Dias MN (1996): Erythrocyte spectrin maintains its segmental motions on oxidation: A Spinlabel EPR study. Biophys. J. 70, 841-851 https://doi.org/10.1016/S0006-3495(96)79626-1

Gabriel S, Lau RW, Gabriel C (1996): The dielectric properties of biological tissues: II. Measurements in the frequency range 10 Hz to $20 \mathrm{GHz}$. Phys. Med. Biol. 41, 2251-2269 https://doi.org/10.1088/0031-9155/41/11/002

Georgatos SD, Marchesi VT (1985): The binding of vimentin to human erythrocyte membranes: a model system for the study of intermediate filament-membrane interactions. J. Cell Biol. 100, 1955-1961 https://doi.org/10.1083/jcb.100.6.1955

Gimsa J (2017): Electric and magnetic fields in cells and tissues. In: Reference Module in Materials Science and Materials Engineering (Ed. S. Hashmi), pp. 1-10, Oxford, Elsevier https://doi.org/10.1016/B978-0-12-803581-8.00982-6

Glomski CA, Pica A (2011): The avian erythrocyte: Its Phylogenic Odyssey. CRC Press, Science Publishers, Tylor \& Francis Group. 23-31

Granger BL, Repasky EA, Lazarides E (1982): Synemin and vimentin are components of intermediate filaments in avian erythrocytes. J. Cell Biol. 92, 299-312 https://doi.org/10.1083/jcb.92.2.299

Hayashi Y, Oshige I, Katsumoto Y, Omori S, Yasuda A, Asami K (2008): Dielectric inspection of erythrocyte morphology. Phys. Med. Biol. 53, 2553-2564 https://doi.org/10.1088/0031-9155/53/10/007

Ivanov IT, Paarvanova BK (2016): Dielectric relaxations on erythrocyte membrane as revealed by spectrin denaturation. Bioelectrochemistry 110, 59-68 https://doi.org/10.1016/j.bioelechem.2016.03.007

Ivanov IT, Paarvanova BK (2019a): Thermal dielectroscopy study on the vertical and horizontal interactions in erythrocyte submembrane skeleton. Electrochimica Acta 317, 289-300 https://doi.org/10.1016/j.electacta.2019.05.159

Ivanov IT, Paarvanova BK (2019b): Effect of permeant cryoprotectants on membrane skeleton of erythrocytes. Probl. Cryobiol. Cryomed. 29, 237-245 https://doi.org/10.15407/cryo29.03.237
Ivanov IT, Paarvanova BK, Ivanov V, Smuda K, Baumler H, Georgieva R (2017): Effects of heat and freeze on isolated erythrocyte submembrane Skeletons. Gen. Physiol. Biophys. 36, 155-165 https://doi.org/10.4149/gpb_2016046

Ivanov IT, Popov BK (1993): Do changes in cell shape affect suspension conductivity? Gen. Physiol. Biophys. 12, 311-315

Johnson RM, Taylor G, Meyer DB (1980): Shape and volume changes in erythrocyte ghosts and spectrin-actin networks. J. Cell Biol. 86, 371-376 https://doi.org/10.1083/jcb.86.2.371

Hanai T, Asamia K, Korzumi N (1979): Dielectric theory of concentrated suspensions of Shell-Spheres in particular reference to the analysis of biological cell suspensions. Bull. Inst. Chem. Res., Kyoto Univ. 57, 297-305

Kim S, Magendantz M, Katz W, Solomon F (1987): Development of a differentiated microtubule structure: formation of the chicken erythrocyte marginal band in vivo. J. Cell Biol. 104, 51-59 https://doi.org/10.1083/jcb.104.1.51

Klösgen B, Rümenapp C, Gleich B (2011): Bioimpedance spectroscopy. In BetaSys: Systems Biology of Regulated Exocytosis in Pancreatic ß-Cells (Ed. S. Choi), pp. 241-271, New York, Springer Publishing Company https://doi.org/10.1007/978-1-4419-6956-9_11

Lange Y, Hadesman RA, Steck TL (1982): Role of reticulum in the stability and shape of the isolated human erythrocyte membrane. J. Cell Biol. 92, 714-721 https://doi.org/10.1083/jcb.92.3.714

Lisin R, Ginzburg BZ, Schlesinger M, Feldman Y (1996): Time domain dielectric spectroscopy study of human cells. I. Erythrocytes and ghosts. Biochim. Biophys. Acta 1280, 34-40 https://doi.org/10.1016/0005-2736(95)00266-9

Liu SC, Derick LH, Agre P, Palek J (1990): Alteration of the erythrocyte membrane skeletal ultrastructure in hereditary spherocytosis, hereditary elliptocytosis, and pyropoikilocytosis. Blood 76, 198-205 https://doi.org/10.1182/blood.V76.1.198.198

Marchesi VT (2008): The relevance of research on red cell membranes to the understanding of complex human disease: A personal perspective. Ann. Rev. Pathol. 3, 1-9

https://doi.org/10.1146/annurev.pathmechdis.3.121806.154321

Martinsen OG, Grimnes S, Schwan HP (2002): Interface phenomena and dielectric properties of biological tissue. In: Encyclopedia of Surface and Colloid Science Anonymous (Ed. P. Somasundaran), pp. 2643-2652, Marcel Dekker Inc.

McGough AM, Josephs R (1990): On the structure of erythrocyte spectrin in partially expanded membrane skeletons. Proc. Natl. Acad. Sci. USA 87, 5208-5212 https://doi.org/10.1073/pnas.87.13.5208

Miller M, Solomon F (1984): Kinetics and intermediates of marginal band formation: evidence for peripheral determinants of microtubule organization. J. Cell Biol. 99, 70-75 https://doi.org/10.1083/jcb.99.1.70s

Mosior M, Bobrowska M, Gomułkiewicz J (1990): Effect of the level of ATP and of the state of spectrin on osmotic properties of bovine erythrocytes. Biochim. Biophys. Acta 1022, 355-360 https://doi.org/10.1016/0005-2736(90)90285-V 
Paarvanova B, Slavov T, Ivanov V, Ivanov I (2014): Thermal dielectroscopy study of submembrane spectrin network in animal erythrocytes. Bulgarian Journal of Veterinary Medicine 17, 165-172

Prodan E, Prodan E, Miller JH Jr (2008): The dielectric response of spherical live cells in suspension: an analytic solution. Biophys. J. 95, 4174-4182

https://doi.org/10.1529/biophysj.108.137042

Salbreux G, Charras G, Paluch, E (2012): Actin cortex mechanics and cellular morphogenesis. Trends Cell Biol. 22, 536-545 https://doi.org/10.1016/j.tcb.2012.07.001

Saldanha C, de Oliveira S (2010): An overview about erythrocyte membrane. Clin. Hemorheol. Microcirc. 44, 63-74 https://doi.org/10.3233/CH-2010-1253

Schwan HP (1981): Dielectric properties of biological tissue and physical mechanisms of electromagnetic field interaction. In: Biological Effects of Nonionizing Radiation, ACS Symposium Series 157, (Ed. K.H. Illinger), American Chemical Society, Washington, DC https://doi.org/10.1021/bk-1981-0157.ch008

Schwan HP, Takashima S (1993): Electrical conduction and dielectric behavior in biological systems. Encycl. Appl. Phys. 5, 177-200

Shotton DM, Burke BE, Branton D (1979): The molecular structure of human erythrocyte spectrin: biophysical and electron microscopic studies. J. Mol. Biol. 131, 303-329 https://doi.org/10.1016/0022-2836(79)90078-0

Simeonova M, Wachner D, Gimsa J (2002): Cellular absorption of electric field energy: influence of molecular properties of the cytoplasm. Bioelectrochemistry 56, 215-218 https://doi.org/10.1016/S1567-5394(02)00010-5

Speicher DW (1986): The present status of erythrocyte spectrin structure: the 106-residue repetitive structure is a basic feature of an entire class of proteins. J. Cell. Biochem. 30, $245-258$

https://doi.org/10.1002/jcb.240300306
Speicher DW, Marchesi VT (1984): Erythrocyte spectrin is comprised of many homologous triple helical segments. Nature 311, 177-180 https://doi.org/10.1038/311177a0

Trinczek B, Marx A, Mandelkow EM, Murphy DB, Mandelkow E (1993): Dynamics of microtubules from erythrocyte marginal bands. Mol. Biol. Cell 4, 323-335

https://doi.org/10.1091/mbc.4.3.323

Tse WT, Lux SE (1999): Red blood cell membrane disorders. Br. J. Haematol. 104, 2-13 https://doi.org/10.1111/j.1365-2141.1999.01130.x

Wasenius VM, Saraste M, Knowles J, Virtanen 1, Letlm VP (1985): Sequencing of the chicken nonerythroid spectrin cDNA reveals an internal repetitive structure homologous to the human erythrocyte spectrin. EMBO J. 4, 1425-1430 https://doi.org/10.1002/j.1460-2075.1985.tb03797.x

Waugh R, Evans EA (1976): Viscoelastic properties of erythrocyte membranes of different vertebrate animals. Microvasc Res. 12, 291-304 https://doi.org/10.1016/0026-2862(76)90027-3

Whitfield CF, Mylin LM, Goodman SR (1983): Species-dependent variations in erythrocyte membrane skeletal proteins. Blood 61, 500-506 https://doi.org/10.1182/blood.V61.3.500.500

Windberger U, Bartholovitsch A, Plasenzotti R, Korak KJ, Heinze G (2003): Whole blood viscosity, plasma viscosity and erythrocyte aggregation in nine mammalian species: reference values and comparison of data. Exp. Physiol. 88, 431-440 https://doi.org/10.1113/eph8802496

Wolf M, Gulich R, Lunkenheimer P, Loidl A (2011): Broadband dielectric spectroscopy on human blood. Biochim. Biophys. Acta 1810, 727-740 https://doi.org/10.1016/j.bbagen.2011.05.012

Received: November 4, 2019

Final version accepted: September 3, 2020 